\title{
Editorial
}

\section{Applied Mathematics and Algorithms for Cloud Computing and IoT}

\author{
Yuxin Mao, ${ }^{1}$ Vijay Bhuse, ${ }^{2}$ Zhongmei Zhou, ${ }^{3}$ Pit Pichappan, ${ }^{4}$ \\ Mahmoud Abdel-Aty, ${ }^{5}$ and Yoshinori Hayafuji ${ }^{6}$ \\ ${ }^{1}$ School of Computer and Information Engineering, Zhejiang Gongshang University, Hangzhou 310018, China \\ ${ }^{2}$ Department of Computing, East Tennessee State University, Johnson City, TN 37614-1266, USA \\ ${ }^{3}$ Department of Computer Science and Engineering, Zhangzhou Normal University, Zhangzhou, China \\ ${ }^{4}$ School of Information Systems, Al Imam University, Riyadh, Saudi Arabia \\ ${ }^{5}$ Scientific Publishing Center, Bahrain University, 32038 Sakhir, Bahrain \\ ${ }^{6}$ Graduate School of Science and Technology, Kwansei Gakuin University, 2-1 Gakuen, Sanda, Hyogo 669-1337, Japan
}

Correspondence should be addressed to Yuxin Mao; maoyuxin@zjgsu.edu.cn

Received 8 January 2014; Accepted 8 January 2014; Published 13 February 2014

Copyright (C) 2014 Yuxin Mao et al. This is an open access article distributed under the Creative Commons Attribution License, which permits unrestricted use, distribution, and reproduction in any medium, provided the original work is properly cited.

Today's world is drifting in all new-fangled technology revolution due to the influence of cloud computing and Internet of Things (IoT) technologies. Currently, cloud computing and IoT are the hottest issues of future Internet.

Cloud computing provides a model for enabling convenient, on-demand network access to a shared pool of configurable computing resources that can be rapidly provisioned and released with minimal management effort or service provider interaction [1]. Cloud computing can be treated as a long-held dream of computing as a utility and has the potential to transform a large part of the IT industry, shaping the way IT hardware is designed and purchased [2]. The term Internet of Things (IoT) was proposed by Kevin Ashton in 1999 [3].

As another emerging research field, IoT draws up a novel paradigm that is rapidly gaining ground in the scenario of modern wireless telecommunications [4]. The major strength of the IoT idea is the high impact it will bring on several aspects of everyday life and behavior of potential users. The research of IoT is still in its infancy. Therefore, there are not any standard definitions for Internet of Things [5].

We experience altogether a different capability when these two technologies go hybrid. There are plenty of new tools and applications coming up in a day-to-day manner in these two fields for our life. However, there are many complex real-life problems (especially engineering problems) in these fields, which require us to solve by using mathematical methods and efficient algorithms. We shall think about these new challenges from the points of both mathematics and computers.

The papers in this special issue cover a wide range of topics in cloud computing and IoT, from underlying infrastructure and algorithms to high-level systems and applications.

The term cloud computing is mostly used to sell hosted services in the sense of application service provisioning at a remote location. At the foundation of cloud computing is the broader concept of shared services and converged infrastructure. Therefore, service is obviously a very important issue in cloud computing (also for IoT). In this special issue, several papers are devoted to this topic. All these papers try to use some kind of mathematical methods to solve the serviceoriented problem in cloud computing.

As the major focus of IoT, equipping all objects in the world with minuscule identifying devices or machine-readable identifiers could transform our daily life $[6,7]$. IoT technologies can be used in a wide range of applications. A number of papers in this special issue have shown their research efforts on using mathematic methods to build IoT applications in smart grid, industry monitoring, city emergency, and so forth. 
Intelligent or optimization algorithms are widely used in both cloud computing and IoT. A dozen of papers in this issue just talk about how to use existing intelligent algorithms, such as classification, self-learning, evidence theory, predicting, evolutionary algorithms, and fuzzy logic, to solve the problems in cloud computing and IoT.

Security is another basic issue for both cloud computing and IoT. Either cloud computing or IoT has a feature of openness in applications. Therefore, how to preserve information security is very important to cloud computing and IoT. The research of information security or network security has an inborn relationship with mathematics. Some papers in this issue also focus on this problem.

Social networking service (SNS) or social media [8], as high-level applications based on cloud computing, have become more and more important in our daily life. SNS also has a close connection to IoT, as many SNS applications are built upon mobile networks. Therefore, how to analyze those large-scale SNS is an interesting topic for both cloud computing and IoT, which relies on quantitive or numerical approaches.

We hope that readers will find in this special issue not only the new ideas, cutting-edge information, new technologies, and applications of cloud computing and IoT, but also a special emphasis on how to solve various engineering problems by using applied mathematics and algorithms.

\author{
Yuxin Mao \\ Vijay Bhuse \\ Zhongmei Zhou \\ Pit Pichappan \\ Mahmoud Abdel-Aty \\ Yoshinori Hayafuji
}

\title{
References
}

[1] P. Mell and T. Grance, "The NIST definition of cloud computing," 2009, http://csrc.nist.gov/publications/nistpubs/800-145/ SP800-145.pdf.

[2] M. Armbrust, A. Fox, R. Griffith et al., "A view of cloud computing," Communications of the ACM, vol. 53, no. 4, pp. 50-58, 2010.

[3] A. Kevin, "That "Internet of Things" Thing, in the real world things matter more than ideas," RFID Journal, June 2009.

[4] L. Atzori, A. Iera, and G. Morabito, "The Internet of Things: a survey," Computer Networks, vol. 54, no. 15, pp. 2787-2805, 2010.

[5] C. Perera, A. Zaslavsky, P. Christen, and D. Georgakopoulos, "Context aware computing for the internet of things: a survey," IEEE Communications Surveys \& Tutorials, vol. 16, no. 1, pp. 414-454, 2014.

[6] P. Magrassi, A. Panarella, N. Deighton, and G. Johnson, "Computers to acquire control of the physical world," Gartner Research Report T-14-0301, 2001.

[7] C. Associati, “The evolution of Internet of Things," 2011, http:// www.casaleggio.it/pubblicazioni/Focus_internet_of_things_ v1.81\%20-\%20eng.pdf.

[8] D. M. Boyd and N. B. Ellison, "Social network sites: definition, history, and scholarship," Journal of Computer-Mediated Communication, vol. 13, no. 1, pp. 210-230, 2007. 


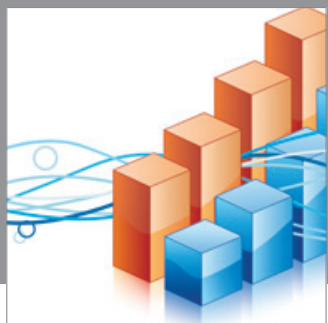

Advances in

Operations Research

mansans

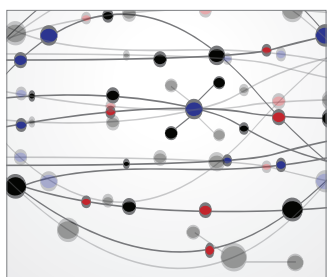

The Scientific World Journal
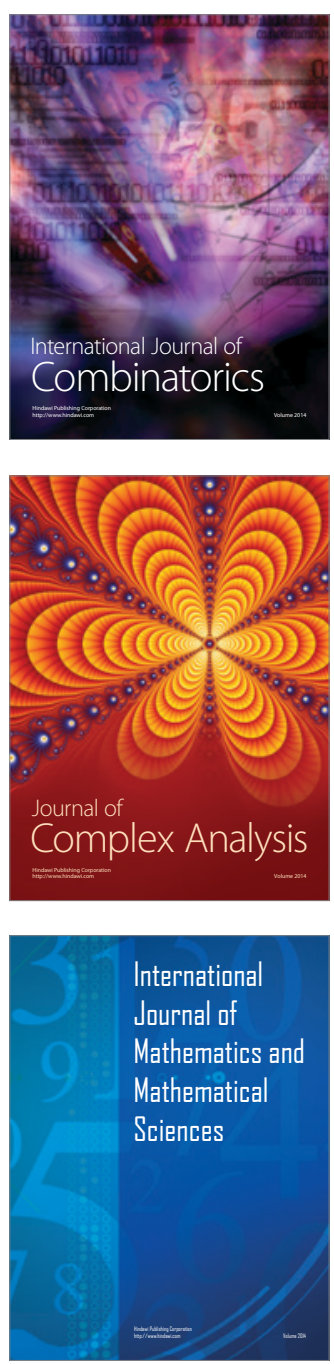
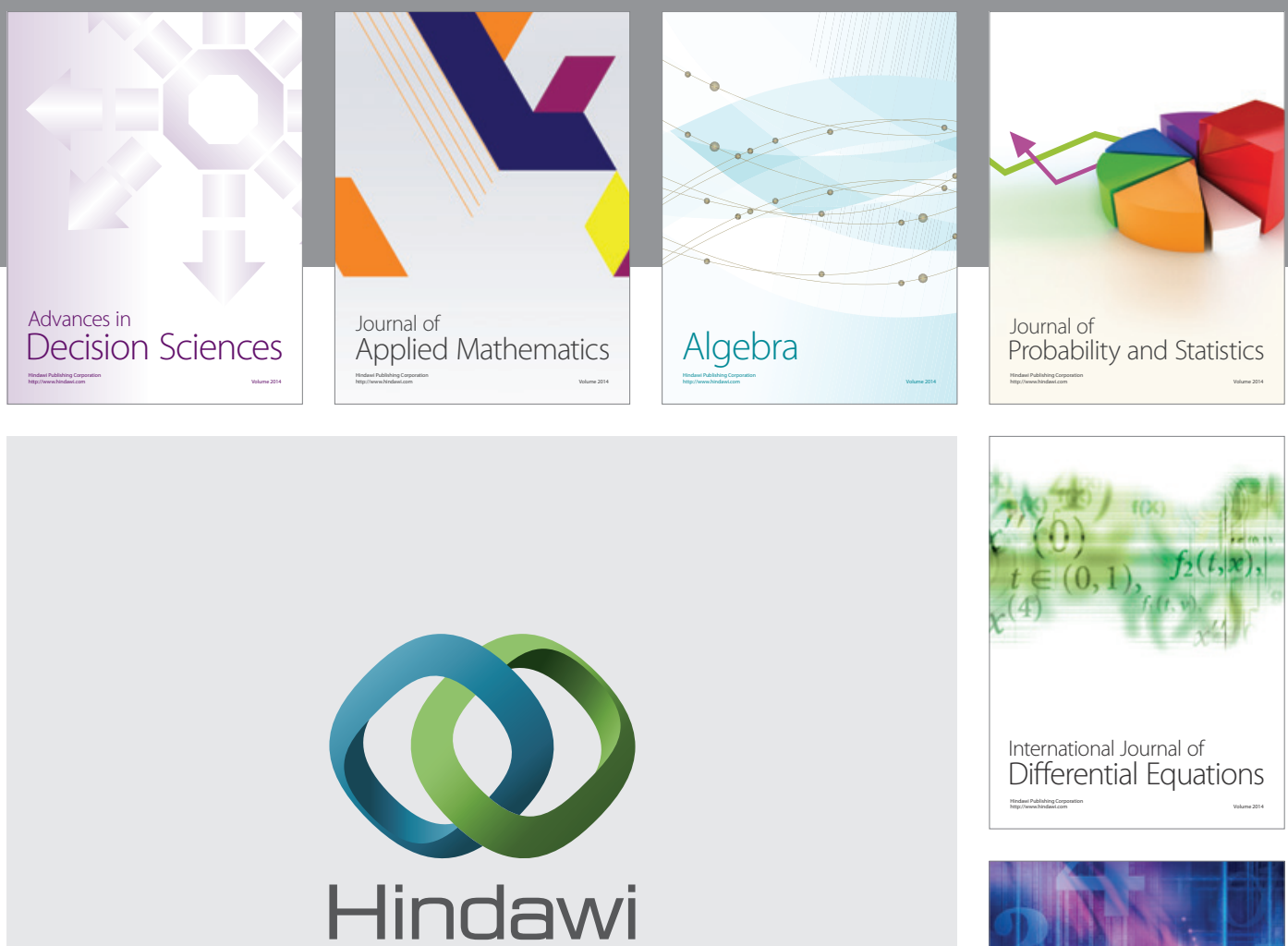

Submit your manuscripts at http://www.hindawi.com
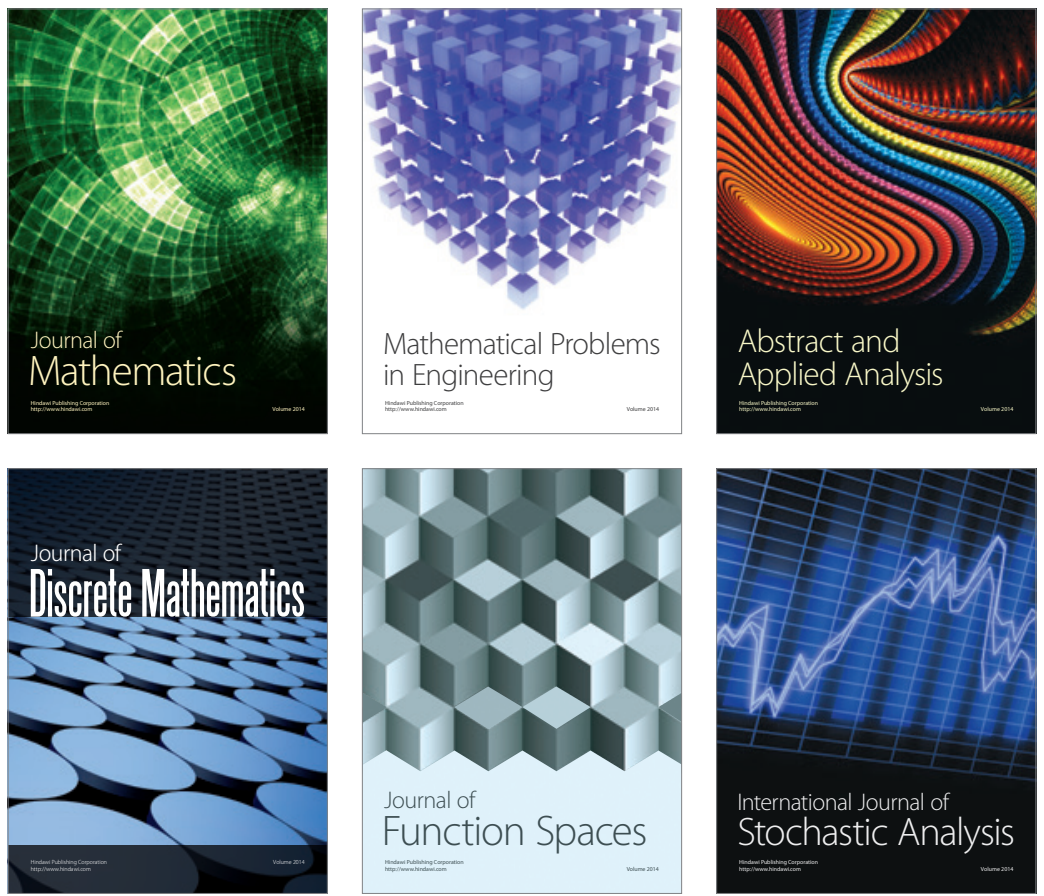

Journal of

Function Spaces

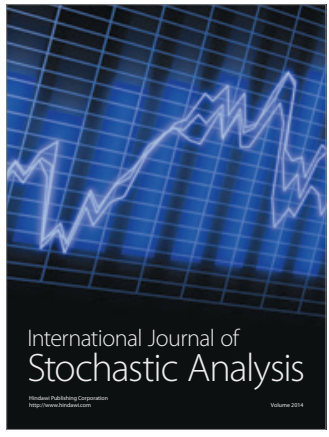

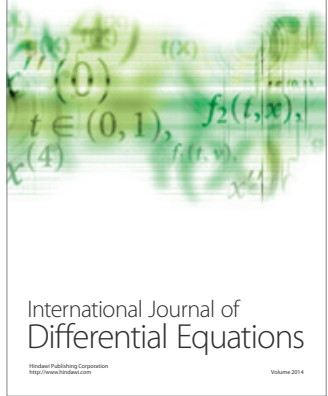
